\title{
Comparison of glaucoma-diagnostic ability between wide-field swept-source OCT retinal nerve fiber layer maps and spectral- domain OCT
}

\author{
Won June Lee ${ }^{1} \cdot$ Sohee $\mathrm{Oh}^{2} \cdot$ Young Kook $\mathrm{Kim}^{3,4} \cdot$ Jin Wook Jeoung ${ }^{3,4} \cdot \mathrm{Ki}$ Ho Park $\mathbb{1}^{3,4}$ \\ Received: 17 May 2017 / Revised: 5 February 2018 / Accepted: 8 February 2018 / Published online: 23 May 2018 \\ (c) The Royal College of Ophthalmologists 2018
}

\begin{abstract}
Purpose To compare the diagnostic ability of wide-field swept-source optical coherence tomography (SS-OCT) retinal nerve fiber layer (RNFL) maps with spectral-domain OCT (SD-OCT) maps for detection of preperimetric (PPG) and early glaucoma (EG).

Patients and methods One hundred and forty-six eyes, including 37 healthy eyes, 38 eyes with PPG, and 71 eyes with EG, were analyzed. The patients underwent both SD-OCT (Cirrus HD-OCT; Carl Zeiss Meditec, Dublin, CA, USA) and widefield SS-OCT scanning (DRI-OCT-1 Atlantis; Topcon, Tokyo, Japan). By SD-OCT, circumpapillary RNFL and macular ganglion cell analyses were performed. SS-OCT provides a wide-field RNFL thickness map and a SuperPixel map, which are composed of an RNFL deviation map of the peripapillary area and a deviation map of the composition of the ganglion cell layer with the inner plexiform layer and RNFL [GC-IPL+RNFL] in the macular area. The ability to discriminate PPG and EG from healthy eyes was assessed according to sensitivity, specificity and area under the receiver operating characteristic curve for parameters and criteria provided by SD-OCT and wide-field SS-OCT scanning.

Results The wide-field RNFL thickness map obtained by SS-OCT showed the highest sensitivity to PPG and EG (92.1 and $97.2 \%$, respectively) as compared with the other, SD-OCT criteria. The wide-field RNFL thickness map showed PPGdiagnostic performance comparable to the SD-OCT RNFL thickness and GC-IPL deviation maps ( $p=0.453$ and 0.180 ), and PPG-diagnostic performance superior to the SD-OCT RNFL deviation and GC-IPL thickness maps $(p=0.003$ and 0.039$)$. In EG, the wide-field RNFL thickness and SuperPixel maps showed diagnostic performance comparable to the SD-OCT thickness and deviation maps ( $p=0.065$ to 0.100$)$, except for the GC-IPL thickness map $(p=0.004)$.

Conclusions The wide-field SS-OCT RNFL thickness maps showed a diagnostic ability for distinguishing PPG and EG from healthy eyes that was similar to that of SD-OCT. In the clinical setting, these maps can be effective for detection of earlyglaucomatous changes.
\end{abstract}

Electronic supplementary material The online version of this article (https://doi.org/10.1038/s41433-018-0104-5) contains supplementary material, which is available to authorized users.

Ki Ho Park

kihopark@snu.ac.kr

1 Department of Ophthalmology, Hanyang University Hospital, Hanyang University College of Medicine, Seoul, Korea

2 Department of Biostatistics, Seoul National University Boramae Hospital, Seoul, Korea

3 Department of Ophthalmology, Seoul National University College of Medicine, Seoul, Korea

4 Department of Ophthalmology, Seoul National University Hospital, Seoul, Korea

\section{Introduction}

Glaucoma is an optic neuropathy characterized by retinal ganglion cell (RGC) degeneration that leads to visual field (VF) loss [1]. Reduction of retinal nerve fiber layer (RNFL) thickness is an early sign of glaucoma, and a significant reduction of RGCs can occur before VF defect is detected [2-7]. Thankfully however, the recent evolution of optical coherence tomography (OCT) has made possible the quantitative measurement of RNFL and macular RGC thicknesses and analysis and comparison with an internal normative database. Indeed, many spectral-domain OCT (SD-OCT) studies have reported diagnostic abilities for glaucoma detection [8-10]. The conventional, widely utilized parameters for OCT-based glaucoma diagnosis are 
those of circumpapillary RNFL (cpRNFL) measurement, though recent studies have reported comparable glaucomadetection ability for macular RGC analysis [11-16]. Also, the SD-OCT RNFL thickness and deviation maps have been reported to have good glaucoma-detection ability [17-20]. More recently, swept-source OCT (SS-OCT), with its advantages in speed and wavelength (for deeper penetration) relative to SD-OCT, has been introduced [21, 22] and employed in the glaucoma field for imaging of deep ocular structures such as the lamina cribrosa we well as for widefield visualization of the posterior pole including the optic nerve head and macula with one, single-scan protocol [23-25]. The glaucoma-diagnostic ability of wide-field-SSOCT-based cpRNFL thickness and macular ganglion cellinner plexiform layer (GC-IPL) measurements has been successfully demonstrated: its wide-field scanning was proved effective for detection of glaucomatous damage, and its diagnostic accuracy was shown to be comparable to that of SD-OCT [26, 27]. More recently, our group reported a study demonstrating the superior glaucoma-diagnostic ability of wide-field SS-OCT RNFL thickness maps to the conventional criteria, as based on the internal normative database [28]. However, to our knowledge, no studies have compared the diagnostic performance of wide-field SS-OCT RNFL maps with conventional SD-OCT for detection of early-stage glaucoma.

Therefore, the present study was designed to compare the ability of wide-field SS-OCT RNFL maps for detection of early-glaucomatous changes with the SD-OCT conventional method including RNFL and GC-IPL thickness and deviation maps.

\section{Subjects and methods}

\section{Participants}

For this prospective cross-sectional study, we enrolled 146 eyes of 146 subjects: 37 healthy eyes, 38 eyes with preperimetric glaucoma (PPG), and 71 eyes with early glaucoma (EG). All of the participants had visited the Glaucoma Clinic of Seoul National University Hospital from August 2014 through September 2015. The subjects were enrolled for the 'Macular Ganglion Cell Imaging Study', an ongoing prospective study designed in 2011. The study protocol, approved by the Institutional Review Board of Seoul National University Hospital, adhered to the tenets of the Declaration of Helsinki.

Each patient underwent a comprehensive ophthalmic examination, including a medical history review, slit-lamp biomicroscopy, intraocular pressure (IOP) measurement using Goldmann applanation tonometry, gonioscopy, disc examination using a 90-D lens, color fundus and disc photography we well as red-free fundus photography (VX10; Kowa Optimed, Tokyo, Japan), standard automated perimetry 24-2 testing (Humphrey Field Analyzer; Carl Zeiss Meditec, Dublin, CA, USA), SD-OCT (Cirrus HDOCT, Carl Zeiss Meditec), and SS-OCT (DRI-OCT-1 Atlantis, Topcon, Tokyo, Japan).

A normal VF was defined as a mean deviation (MD) and pattern standard deviation (PSD) within 95\% confidence limits and a glaucoma hemifield test (GHT) result within normal limits. Eyes with glaucomatous VF defects were defined as those with a cluster of three points with probabilities of $<5 \%$ on the pattern deviation map in at least 1 hemifield, including at least 1 point with a probability of $<1 \%$; or a cluster of two points with a probability of $<1 \%$, and a GHT result outside $99 \%$ of agespecific normal limits or a PSD outside $95 \%$ of normal limits.

The inclusion criteria were a best-corrected visual acuity of 20/40 or better, spherical equivalent refractive errors between +6.0 and $-6.0 \mathrm{D}$, cylinder correction $<3.0 \mathrm{D}$, and an open anterior chamber angle. All of the SS-OCT images had an image quality score of 50 or better, while all of the SD-OCT images had a signal strength of 7 or better. The exclusion criteria were as follows: a history of ophthalmic surgery (such as glaucoma-filtering surgery), any other macular disease that could interfere with segmentation of retinal layers, and in ability to obtain high-quality OCT image.

Glaucomatous eyes were defined by the presence of a characteristic optic disc (i.e., localized or diffuse neuroretinal rim thinning, increased cupping or cup-to-disc ratio difference $>0.2$ between the eyes) on stereo disc photography or the presence of RNFL defect on red-free fundus imaging, regardless of the presence or absence of glaucomatous VF defects. On the basis of the VF test results, the patients with glaucoma were divided into two groups: PPG (normal VF) and $\mathrm{EG}$ (VF loss with $\mathrm{MD} \geq-6 \mathrm{~dB}$ ). If both of a patient's eyes were glaucomatous, one eye was randomly selected. Healthy control was defined as satisfying all of the following criteria in both eyes: (1) no history or evidence of intraocular surgery, (2) IOP $\leq 21 \mathrm{mmHg}$ with no history of increased IOP, (3) absence of glaucomatous disc appearance, (4) absence of glaucomatous VF defects, and (5) no other significant ophthalmic findings. One healthy eye from healthy control was randomly selected and analyzed in this study.

\section{Optical coherence tomography}

All of the subjects underwent SD-OCT and SS-OCT imaging on the same day, in random order. All of these OCT examinations were performed by one experienced technician. cpRNFL measurement and macular ganglion cell 


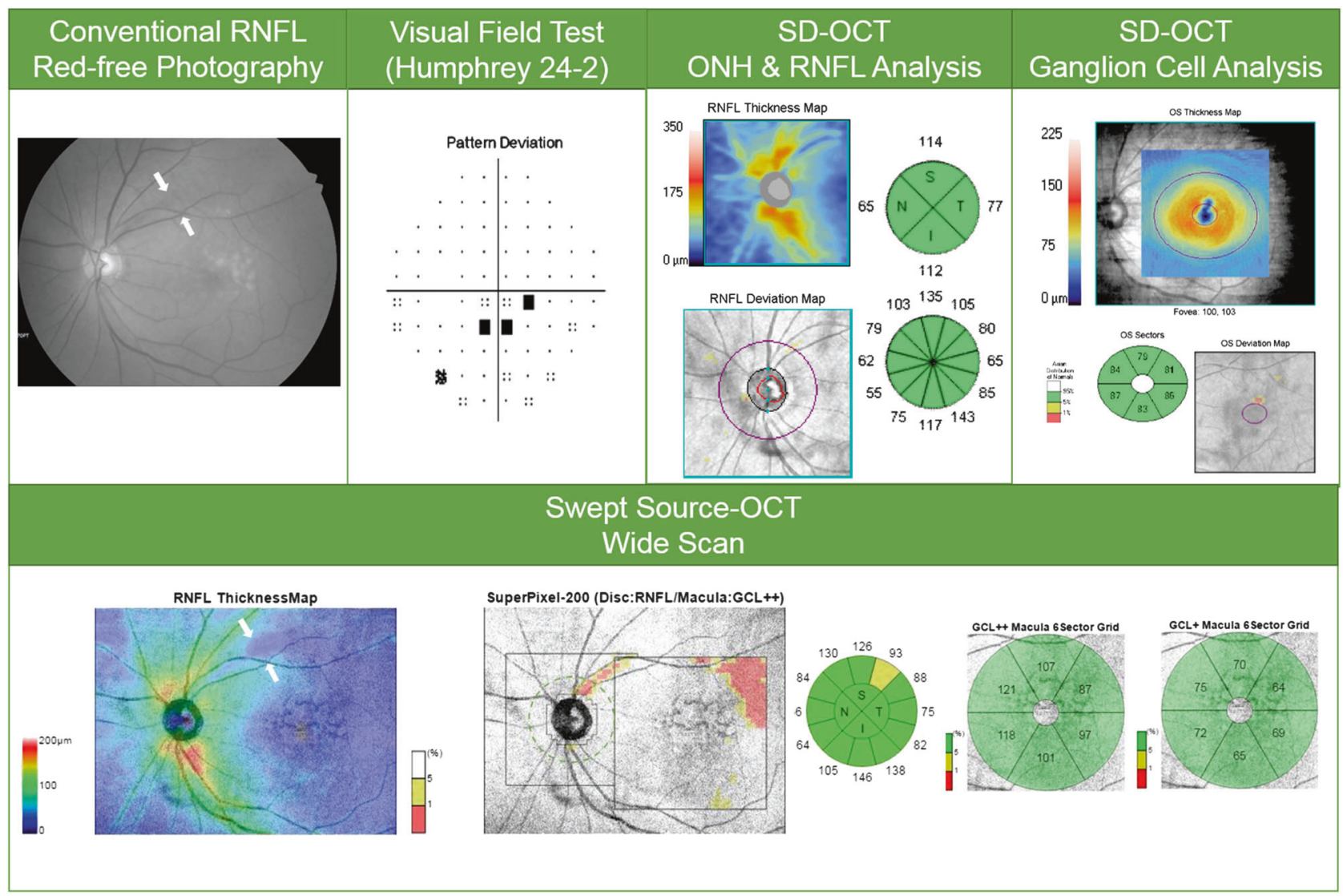

Fig. 1 (Case 1) 76-year-old man with primary open-angle glaucoma in his left eye. Conventional red-free photography revealed a superotemporal RNFL defect (white arrows). cpRNFL analysis and SDOCT macular ganglion cell analysis revealed no abnormality. The wide-field SS-OCT RNFL thickness map clearly showed a wedge-

analysis were performed according to the right-eye orientation.

With regard to SD-OCT, the optic disc cube $200 \times 200$ and macular cube $200 \times 200$ protocols were used to measure cpRNFL and GC-IPL thickness, respectively. With respect to SS-OCT meanwhile, the wide-field scan protocol (with embedded software) was applied to obtain the wide-field RNFL thickness map. Thereby, it was possible to obtain images of the macular and optic nerve head regions in a single scan, for a total acquisition time of only $1.3 \mathrm{~s}$. The $12 \times 9 \mathrm{~mm}$ scan comprised $256 \mathrm{~B}$-scans, each in turn comprising 512 A-scans, for a total of 131,072 axial scans/volume.

With the built-in analysis software (version 9.30), the RNFL boundary was automatically segmented and the RNFL thickness throughout the scan was calculated. The RNFL thickness map was generated within the $12 \times 9 \mathrm{~mm}$ field area with color scales corresponding to the numeric RNFL thickness measurements. Abnormal results based on a comparison of the RNFL and macular [GC-IPL+RNFL] thicknesses to the normative database were displayed on the shaped dark-blue area indicating RNFL thinning (white arrows). Further, the SS-OCT SuperPixel map clearly revealed an arcuate pattern of contiguous abnormal yellow/red pixels extending to the macular area

SuperPixel deviation map. This map typically is composed of a peripapillary RNFL deviation map with $26 \times 26$ pixels within a $5.2 \times 5.2 \mathrm{~mm}^{2}$ peripapillary area and a macular [GCIPL+RNFL] deviation map with $30 \times 30$ pixels within a $6.0 \times 6.0 \mathrm{~mm}^{2}$ macular area. The length of one side in each pixel is $200 \mu \mathrm{m}$. The uncolored pixels indicate the normal range, whereas the yellow-colored or red-colored pixels indicate abnormality at $<5 \%$ and $>1 \%$ or $<1 \%$ of the normal level, respectively (Fig. 1).

This protocol also provides for conventional cpRNFL thickness and macular ganglion cell measurement. SS-OCT segmentation software was used to identify the limits of the RNFL and IPL and to determine the thickness of the cpRNFL, macular GC-IPL, and [GC-IPL+RNFL], throughout the scan. The optic disc center was automatically detected, and a $3.4 \mathrm{~mm}$ diameter circle was drawn around it. Conventional cpRNFL measurements on that circle were taken, and averages were computed for the 4 and 12 o'clock hours. In the macular ganglion cell analysis, GC-IPL thickness included the GC layer and the IPL, and [GC-IPL+RNFL] thickness included the GC-IPL 
and the RNFL. To measure the GC-IPL and [GC-IPL +RNFL] thickness, a circle $6 \mathrm{~mm}$ in diameter was automatically centered on the fovea. Sectoral GC-IPL and [GCIPL + RNFL] thicknesses were measured, and a color-coded map for comparison with the normative database also was displayed.

\section{Definitions of glaucomatous structural changes according to OCT devices}

The definitions of RNFL defect on red-free photography, the wide-field RNFL thickness map and the SuperPixel map have been provided previously and are briefly summarized below [18-20, 24, 28, 29].

\section{Definition of RNFL defect on red-free photography}

RNFL defect on red-free photography was defined as having a width at a 1-disc-diameter distance from the edge of the disc larger than that of a major retinal vessel, and diverging in an arcuate or wedge shape.

\section{Definition of RNFL defect on wide-field RNFL thickness map and the SuperPixel map}

RNFL defect on the wide-field RNFL thickness map was defined as an arcuate or wedge-shaped diverging dark-blue area surrounding an abrupt color-scale change that appeared less thick than neighboring areas on color-coded maps of wide RNFL thickness. The minimum defect size was larger than the diameter of a major retinal vessel.

RNFL defect on the SuperPixel map was arbitrarily defined as the presence of a wedge-shaped area of at least 20 contiguous yellow/red pixels along with RNFL thinning on the deviation map (peripapillary RNFL+Macular [GCIPL+RNFL]).

\section{Definition of glaucomatous structural change on SD-OCT thickness maps}

Glaucomatous structural change on the SD-OCT RNFL thickness map was defined as a wedge-shaped dark-blue area surrounding an abrupt color-scale change. Glaucomatous structural change on the SD-OCT RNFL deviation map was arbitrarily defined as the presence of a wedge-shaped area of at least 20 contiguous yellow/red pixels along with RNFL thinning. On both maps, the width of defect increasing from the disc toward the edge of the map, and the minimum defect size was larger than the diameter of a major retinal vessel.

Glaucomatous structural change on the SD-OCT GC-IPL thickness map was defined as the presence of a blue/black area. Glaucomatous structural change on the SD-OCT GC-
IPL deviation map was defined as the presence of an area of $>10$ pixels size as coded in yellow/red.

Glaucomatous structural changes on the SD-OCT combined (cpRNFL+macular GC-IPL) thickness map and deviation map were determined independently by two glaucoma specialists in a masked fashion.

RNFL defect on red-free photography and both SD-OCT and wide-field SS-OCT thickness maps and deviation maps was evaluated independently by two glaucoma specialists (WJL, YKK) in a masked fashion. For prevention of bias, the graders who evaluated the wide-field RNFL thickness maps were masked to the red-free photographs. When the two experts disagreed, a third ophthalmologist (KHP) evaluated the photographs, and the disagreement was resolved after discussion.

\section{Statistical analysis}

To evaluate the agreement between the SD-OCT and SSOCT thickness values, Bland-Altman plots with regression analysis were used [30]. To assess the abilities of each of the categorical variables such as wide-field RNFL thickness map or the criteria on the basis of a comparison of measurements with the built-in normative database to distinguish subjects with glaucoma from healthy controls, sensitivities and specificities were tested. The comparison between the qualitative variables including sensitivities or specificities was performed using the McNemar test. The area under the receiver operating characteristic curve (AUC) was calculated for continuous parameters such as cpRNFL thickness. The sensitivities at fixed specificities (80 and $90 \%$ ) for each parameter also were calculated. The AUCs, sensitivities at fixed specificities, and the sensitivities and specificities independently were analyzed in PPG and EG eyes, respectively.

A $P$-value of $<0.05$ was considered to indicate significance. All of the statistical tests were performed using PASW Statistics 18 (SPSS, Chicago, IL), and MedCalc (MedCalc Software, Ostend, Belgium).

\section{Results}

A total of 154 patients were enrolled in this study after applying the inclusion criteria. Of these, three patients $(1.9 \%)$ of wet age-related macular degeneration, four patients $(2.6 \%)$ of epiretinal membrane, and one patient $(0.6 \%)$ of branch retinal vein occlusion were excluded. Ultimately, we studied 146 eyes of 146 patients: 37 healthy eyes, 38 eyes with PPG, and 71 eyes with EG. The patient demographics are presented in Supplementary Table 1. Among the three groups, the differences in age, IOP and spherical equivalent were not significant, though those in 
Table 1 Agreement and correlation between cpRNFL and GC-IPL measurements obtained using SS-OCT and SDOCT (SS-OCT-SD-OCT)

\begin{tabular}{|c|c|c|c|c|c|c|c|c|c|}
\hline \multicolumn{5}{|c|}{ Difference between $X$ and $Y(X-Y)$} & \multirow{2}{*}{\multicolumn{5}{|c|}{$\begin{array}{l}\text { Bland-Altman plots regression between }(X+Y) / 2 \\
\text { and }(X-Y)\end{array}$}} \\
\hline \multicolumn{5}{|c|}{$X=$ SS-OCT Result, $Y=$ SD-OCT Result } & & & & & \\
\hline & $\begin{array}{l}\text { Mean } \\
\text { difference }\end{array}$ & $95 \%$ & & $\overline{p \text {-value }}$ & \multicolumn{2}{|l|}{ Fixed bias } & Proportional bias & $p$-value & $R^{2}$ \\
\hline \multicolumn{10}{|l|}{$c p R N F L$} \\
\hline Average & 13.0 & 12.0 & 13.9 & $<0.001$ & -11.3 & $\mathrm{Y}$ & 0.27 & $<0.001$ & 0.37 \\
\hline 12 & 14.1 & 11.8 & 16.5 & $<0.001$ & 2.4 & $\mathrm{~N}$ & 0.11 & 0.013 & 0.04 \\
\hline 1 & 13.9 & 11.6 & 16.2 & $<0.001$ & -9.2 & $\mathrm{~N}$ & 0.23 & $<0.001$ & 0.15 \\
\hline 2 & 7.3 & 5.1 & 9.6 & $<0.001$ & -19.8 & $\mathrm{Y}$ & 0.35 & $<0.001$ & 0.19 \\
\hline 3 & 9.0 & 7.2 & 10.8 & $<0.001$ & -17.0 & $\mathrm{Y}$ & 0.43 & $<0.001$ & 0.13 \\
\hline 4 & 13.9 & 12.0 & 15.8 & $<0.001$ & -24.9 & $\mathrm{Y}$ & 0.59 & $<0.001$ & 0.38 \\
\hline 5 & 18.9 & 16.9 & 20.9 & $<0.001$ & -9.4 & $\mathrm{Y}$ & 0.30 & $<0.001$ & 0.25 \\
\hline 6 & 20.5 & 17.9 & 23.0 & $<0.001$ & -10.3 & $\mathrm{Y}$ & 0.26 & $<0.001$ & 0.30 \\
\hline 7 & 4.0 & 1.6 & 6.4 & 0.001 & -13.7 & $\mathrm{Y}$ & 0.16 & $<0.001$ & 0.18 \\
\hline 8 & 9.1 & 7.8 & 10.4 & $<0.001$ & 10.2 & $\mathrm{Y}$ & -0.02 & 0.751 & 0.00 \\
\hline 9 & 16.0 & 14.9 & 17.2 & $<0.001$ & 10.0 & $\mathrm{Y}$ & 0.10 & 0.155 & 0.02 \\
\hline 10 & 15.9 & 14.8 & 17.0 & $<0.001$ & 7.1 & $\mathrm{Y}$ & 0.10 & 0.001 & 0.08 \\
\hline 11 & 12.9 & 10.9 & 14.9 & $<0.001$ & -8.2 & $\mathrm{Y}$ & 0.18 & $<0.001$ & 0.19 \\
\hline \multicolumn{10}{|l|}{$G C-I P L$} \\
\hline Average & -9.6 & 10.1 & -9.4 & $<0.001$ & 0.31 & $\mathrm{~N}$ & -0.14 & $<0.001$ & 0.18 \\
\hline Superior & -11.2 & 11.9 & -10.4 & $<0.001$ & 8.2 & $\mathrm{Y}$ & -0.27 & $<0.001$ & 0.21 \\
\hline Superonasal & -7.3 & -8.0 & -6.7 & $<0.001$ & 5.6 & $\mathrm{~N}$ & -1.7 & $<0.001$ & 0.09 \\
\hline Inferonasal & -8.0 & -9.1 & -6.8 & $<0.001$ & -0.21 & $\mathrm{~N}$ & -0.11 & 0.007 & 0.05 \\
\hline Inferior & -12.0 & 12.6 & -11.4 & $<0.001$ & 2.20 & $\mathrm{~N}$ & -0.22 & $<0.001$ & 0.25 \\
\hline Inferotemporal & -9.0 & -9.5 & -8.4 & $<0.001$ & -6.4 & $\mathrm{Y}$ & -0.04 & 0.149 & 0.02 \\
\hline Superotemporal & -9.4 & -9.9 & -8.9 & $<0.001$ & -4.5 & $\mathrm{~N}$ & -0.07 & 0.058 & 0.03 \\
\hline
\end{tabular}

$c p R N F L$ circumpapillary RNFL, GC-IPL ganglion cell-inner plexiform layer
MD, PSD, and VF index were (healthy and PPG vs. EG, $p<0.001)$.

\section{Inter-device agreement}

Bland-Altman plots with regression analysis were used to compare the sector thickness values between the two OCT devices. Table 1 summarizes the results. Although most of the values were within the limits of agreement, there were significant proportional biases. Figure 2 shows two representative Bland-Altman plots with significant correlation (Fig. 2a for cpRNFL average, and Fig. 2b for GC-IPL average).

\section{Diagnostic ability to discriminate between PPG and healthy eyes}

The sensitivities and specificities of the following categorical variables provided by the built-in normative database for discrimination between PPG and healthy eyes are presented in Table 2: wide-field RNFL thickness map, wide- field SuperPixel map, SD-OCT RNFL, and GC-IPL thickness and deviation maps, as well as SD-OCT cpRNFL and GC-IPL based on abnormality criteria (color-coded map, $<5 \%$ level or $<1 \%$ level). Among the listed sensitivity values, the highest was that for the wide-field SS-OCT RNFL thickness map (92.1\%). This sensitivity was comparable to those of the SD-OCT RNFL thickness map, GC-IPL deviation map, and RNFL+GC-IPL thickness map ( $p=0.453,0.180$, and 0.687 , respectively) and was significantly higher than that of the SD-OCT RNFL deviation map, GC-IPL thickness map, and RNFL+GC-IPL deviation map ( $p=0.003,0.039$, and 0.021 , respectively). The sensitivities of the wide-field SS-OCT RNFL thickness map and SuperPixel map were significantly higher than those of the SD-OCT categorical variables with color-coded map, including cpRNFL clock hours and quadrants or GC-IPL sectoral analyses $(p<0.001$ to 0.021$)$.

The AUCs in terms of the quantitative SD-OCT measurement for healthy and PPG eyes are presented in Supplementary Table 2. Among the various SD-OCT parameters, 7 o'clock cpRNFL thickness $(\mathrm{AUC}=0.852$ ) 


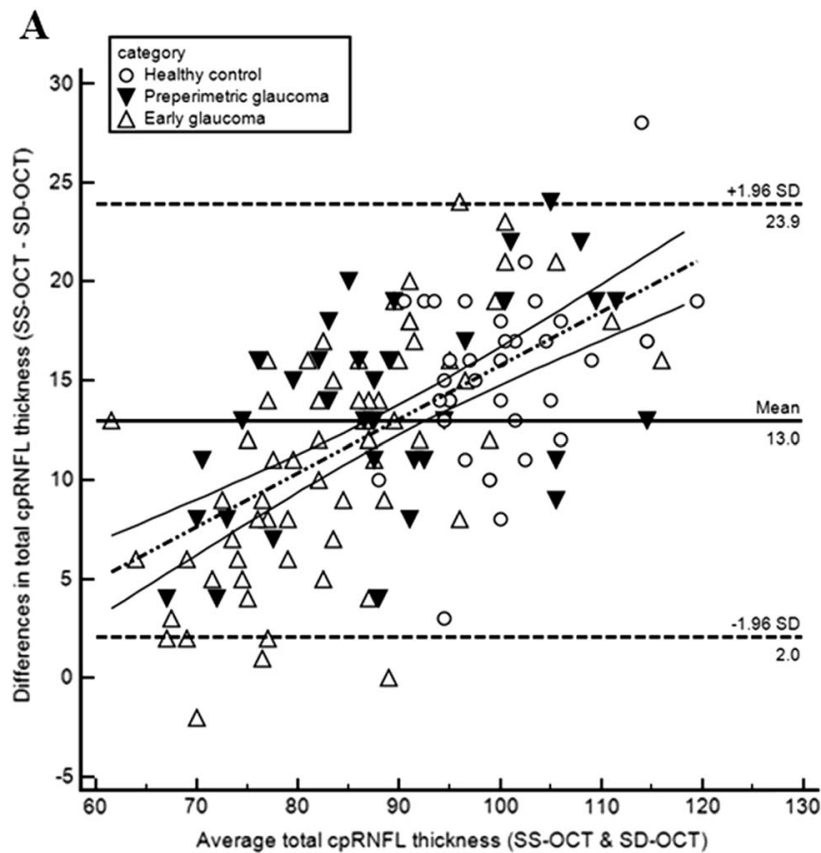

Fig. 2 Bland-Altman plots showing agreement between SS-OCT and SD-OCT. a average thickness of cpRNFL, $\mathbf{b}$ average thickness of GC-

and minimum GC-IPL thickness $(\mathrm{AUC}=0.831)$ showed the best diagnostic performances. All of the SD-OCT parameters' sensitivities at fixed specificities of 80 and $90 \%$ were significantly lower than that of the wide-field SS-OCT RNFL thickness map $(p<0.001$ to $p=0.043$ and $p<0.001$ to $p=0.006$, respectively).

\section{Diagnostic ability to discriminate between EG and healthy eyes}

The sensitivities and specificities of the categorical variables for discrimination between EG and healthy eyes are presented in Table 3 . The two highest sensitivity values were those for the wide-field SS-OCT RNFL thickness (97.2\%) and SuperPixel (93.0\%) maps. These were comparable to those of the SD-OCT thickness and deviation maps (RNFL thickness and deviation maps, GC-IPL deviation map, and RNFL+GC-IPL thickness and deviation maps: $p=0.065$ to 1.000) and significantly higher than that of the GC-IPL thickness map $(p=0.004)$. The sensitivities of these two SS-OCT variables, in fact, were significantly higher than those of most of the other SD-OCT categorical variables with color-coded map, including cpRNFL clock hours and quadrants or GC-IPL sectoral analyses $(p<0.001$ to 0.012$)$.

The AUCs in terms of the quantitative SD-OCT measurement for healthy and EG eyes are presented in Supplementary Table 3. Among the various SD-OCT parameters, total cpRNFL thickness $(\mathrm{AUC}=0.911)$, 7 o'clock cpRNFL thickness (AUC $=0.893)$, and minimum

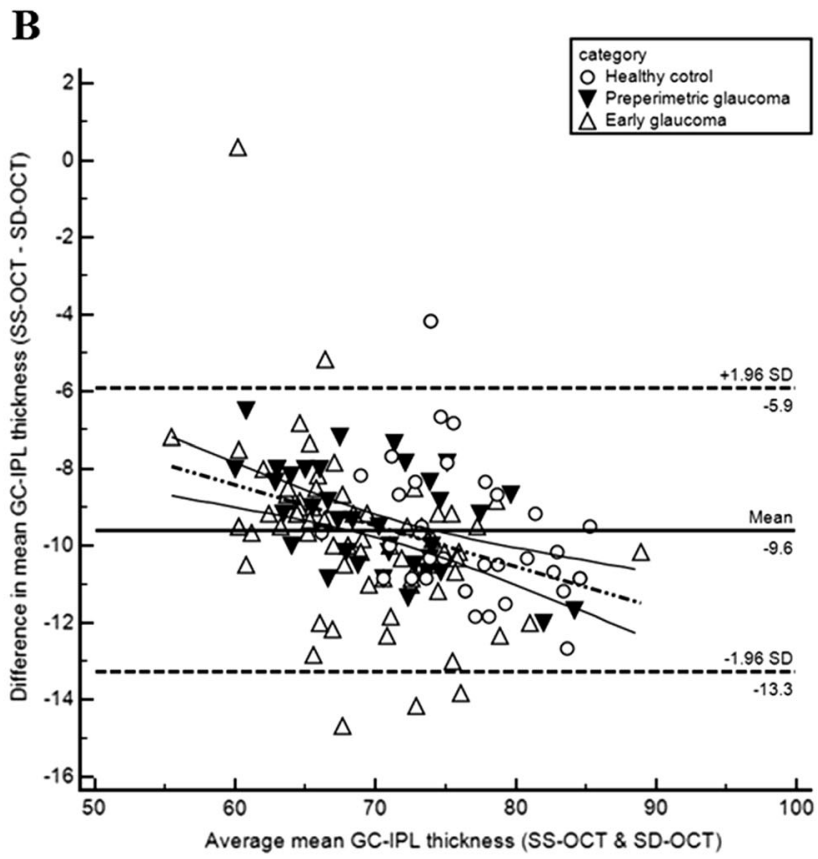

IPL. a Average cpRNFL thickness $(y=-11.3+0.27 x)$. b Average GC-IPL thickness $(y=0.31-0.14 x)$

GC-IPL thickness $(A U C=0.891)$ showed the best diagnostic performances. All of the SD-OCT parameters' sensitivities at fixed specificities of 80 and $90 \%$ were significantly lower than that of the wide-field SS-OCT RNFL thickness map ( $p<0.001$ to $p=0.027$ and $p<0.001$ to $p=0.004$, respectively).

\section{Discussion}

In the present study, we evaluated the diagnostic ability of wide-field SS-OCT RNFL maps to differentiate glaucomatous from healthy eyes and compared them with SD-OCT. To our knowledge, this is the first report to compare the ability of wide-field SS-OCT RNFL maps with the conventional SD-OCT method for detection of earlyglaucomatous damage. Our results indicated that the diagnostic abilities of the wide-field SS-OCT RNFL maps were good and comparable to those of the conventional SD-OCT parameters for discrimination of PPG or EG from healthy eyes.

Among the relevant previous investigations, Lee et al. [23], evaluating SS-OCT/SD-OCT agreement for normal eyes utilized the OCT devices as we did, while Yang et al. [26] evaluated SS-OCT/SD-OCT agreement for healthy and glaucomatous eyes. In both of those studies, cpRNFL thickness was thicker for SS-OCT measurements than for SD-OCT, whereas GC-IPL thickness was thinner for SSOCT than for SD-OCT. These results were consistent with 
Table 2 Sensitivities and specificities for discrimination between preperimetric glaucoma and healthy control (categorical variables-criteria)

\begin{tabular}{lllllll}
\hline Healthy vs. PPG & Sensitivity & $p$-value 1 & $p$-value 2 & Specificity & $p$-value 1 & $p$-value 2 \\
\hline Wide-field RNFL thickness map & 92.1 & & 0.453 & 89.2 & & 1.000 \\
Wide-field SuperPixel map & 84.2 & 0.453 & & 91.9 & 1.000 & \\
SD-OCT & & & & & & \\
RNFL thickness map & 84.2 & 0.453 & 1.000 & 70.3 & 1.000 & 1.000 \\
RNFL deviation map & 63.2 & $\mathbf{0 . 0 0 3}$ & $\mathbf{0 . 0 0 8}$ & 89.2 & 1.000 & 1.000 \\
GC-IPL thickness map & 71.1 & $\mathbf{0 . 0 3 9}$ & 0.227 & 86.5 & 1.000 & 1.000 \\
GC-IPL deviation map & 78.9 & 0.180 & 0.625 & 64.9 & 0.004 & 0.006 \\
Thickness map (RNFL+GC-IPL) & 86.8 & 0.687 & 1.000 & 94.6 & 0.625 & 1.000 \\
Deviation map (RNFL+GC-IPL) & 71.1 & $\mathbf{0 . 0 2 1}$ & 0.063 & 100.0 & N/A & N/A \\
SD-OCT cpRNFL thickness & & & & & & \\
Clock hours <1\% (red) & 47.4 & $\mathbf{4 0 . 0 0 1}$ & $<\mathbf{0 . 0 0 1}$ & 94.6 & 0.687 & 1.000 \\
Quadrants <1\% (red) & 39.5 & $<\mathbf{0 . 0 0 1}$ & $<\mathbf{0 . 0 0 1}$ & 97.3 & 0.375 & 0.625 \\
Clock hours <5\% (yellow) & 65.8 & $\mathbf{0 . 0 0 6}$ & $\mathbf{0 . 0 1 6}$ & 64.9 & 0.012 & $\mathbf{0 . 0 0 2}$ \\
Quadrant <5\% (yellow) & 63.2 & $\mathbf{0 . 0 0 3}$ & $\mathbf{0 . 0 2 1}$ & 91.9 & 1.000 & 1.000 \\
SD-OCT ganglion cell analysis & & & & & & \\
GC-IPL<1\% (red) & 60.5 & $\mathbf{0 . 0 0 2}$ & $\mathbf{0 . 0 0 4}$ & 86.5 & 1.000 & 0.727 \\
GC-IPL<5\% (yellow) & 86.8 & 0.727 & 1.000 & 70.3 & 0.092 & 0.057 \\
\hline
\end{tabular}

$p$-value 1 index compared with wide-field RNFL thickness map (McNemar's test)

$p$-value 2 index compared with wide-field SuperPixel map (McNemar's test)

Bold values indicates statistically significant with $p$-value less than 0.05

$P P G$ preperimetric glaucoma, $R N F L$ retinal nerve fiber layer, $c p R N F L$ circumpapillary RNFL, $G C-I P L$ ganglion cell-inner plexiform layer ours, though there also were some differences. First, Lee et al. used the optic disc scan protocols of both OCTs for cpRNFL analysis, whereas we and Yang et al. used the wide-field SS-OCT scan protocol. Second, with respect to Lee et al., our study included not only normal healthy eyes but also glaucomatous eyes; third, different from Yang et al., our study included PPG eyes; and fourth, regarding Yang et al., our result showed a larger discrepancy in cpRNFL measurement between the two OCT devices. Because our study enrolled only subjects with early stages of glaucoma, cpRNFL thickness did not decrease by considerable amounts. In cases of advanced glaucoma, cpRNFL thickness might have decreased considerably for both OCT devices, which may have resulted in smaller inter-device discrepancy in Yang et al.'s study.

Although most of the presently obtained values were within the limit of agreement identified from Bland-Altman plots, the thickness was not interchangeable between the two OCT devices, because significant proportional bias existed in most sectors. The existence of proportional bias indicates that the methods do not agree equally through the range of measurements, and indeed, there is a significant tendency to disagreement. This result also is consistent with the above-noted previous studies [23, 26]. The reasons for these disagreements, which are two, should be considered. First, the imaging acquisition and analysis protocol are different. SS-OCT analyzes the cpRNFL profile of a $3.4 \mathrm{~mm}$ diameter circle and GC-IPL thickness within a macular 6 $\mathrm{mm}$ diameter circle, whereas SD-OCT analyzes a $3.46 \mathrm{~mm}$ diameter circle for cpRNFL and a macular $4.8 \times 4.0$ elliptical area for GC-IPL measurement. Regarding cpRNFL, more RNFL fibers are collected closer to the optic disc, and so SS-OCT with a smaller circle size shows a thicker cpRNFL. On the other hand, regarding GC-IPL, it becomes relatively thicker closer to the fovea, and so SD-OCT, with its smaller area of analysis, shows thicker values [23]. Second, image distortion due to wide-field scans can occur. Compared with focusing individually on the disc or fovea as in conventional scans, scanning a wide area can cause additional distortion in projecting a curved $3 \mathrm{D}$ surface to 2D. Notably in this regard, the cpRNFL circle, as it is centered on the region between the optic disc and the macula, scans a slanted RNFL thickness around the disc [26].

Several studies have evaluated the glaucoma-diagnostic ability of SS-OCT relative to that of SD-OCT [26, 27, 31, 32]. Two of them reported similar diagnostic ability between SS-OCT RNFL thickness measurement in the macular and peripapillary areas and SS-OCT or Spectralis SD-OCT cpRNFL thickness measurement [27, 31]. Two of the other studies, meanwhile, reported comparable diagnostic abilities for SS-OCT and Cirrus or Spectralis SDOCT in the evaluation of macular GC-IPL thicknesses [26, 32]. Two of the four aforementioned studies, moreover, 
Table 3 Sensitivities and specificities for discrimination between the early glaucoma and healthy control (categorical variables-criteria)

\begin{tabular}{lllllll}
\hline Healthy vs. EG & Sensitivity & $p$-value 1 & $p$-value 2 & Specificity & $p$-value 1 & $p$-value 2 \\
\hline Wide-field RNFL thickness map & 97.2 & & 0.453 & 89.2 & & 1.000 \\
Wide-field SuperPixel map & 93.0 & 0.453 & & 91.9 & 1.000 & \\
SD-OCT & & & & & & \\
RNFL thickness map & 92.9 & 0.453 & 1.000 & 91.9 & 1.000 & 1.000 \\
RNFL deviation map & 88.6 & 0.109 & 0.453 & 89.2 & 1.000 & 1.000 \\
GC-IPL thickness map & 80.0 & $\mathbf{0 . 0 0 4}$ & $\mathbf{0 . 0 0 4}$ & 86.5 & 1.000 & 0.687 \\
GC-IPL deviation map & 88.6 & 0.109 & 0.375 & 64.9 & $\mathbf{0 . 0 0 4}$ & $\mathbf{0 . 0 0 6}$ \\
Thickness map (RNFL+GC-IPL) & 91.4 & 0.289 & 1.000 & 94.6 & 1.000 & 1.000 \\
Deviation map (RNFL+GC-IPL) & 87.1 & 0.065 & 0.219 & 100.0 & N/A & N/A \\
cpRNFL thickness & & & & & & \\
Clock hours <1\% (red) & 71.4 & $<\mathbf{0 . 0 0 1}$ & $<\mathbf{0 . 0 0 1}$ & 94.6 & 0.687 & 1.000 \\
Quadrants <1\% (red) & 68.6 & $<\mathbf{0 . 0 0 1}$ & $<\mathbf{0 . 0 0 1}$ & 97.3 & 0.375 & 0.625 \\
Clock hours <5\% (yellow) & 87.1 & 0.065 & 0.219 & 64.9 & 0.012 & $\mathbf{0 . 0 0 2}$ \\
Quadrant <5\% (yellow) & 80.0 & $\mathbf{0 . 0 0 4}$ & $\mathbf{0 . 0 1 2}$ & 91.9 & 1.000 & 1.000 \\
Macular ganglion cell analysis & & & & & & \\
GC-IPL <1\% (red) & 78.6 & $\mathbf{0 . 0 0 2}$ & $\mathbf{0 . 0 0 6}$ & 86.5 & 1.000 & 0.727 \\
GC-IPL <5\% (yellow) & 88.6 & 0.109 & 0.508 & 70.3 & 0.092 & 0.057 \\
\hline
\end{tabular}

$p$-value 1 index compared with wide-field RNFL thickness map (McNemar's test)

$p$-value 2 index compared with wide-field SuperPixel map (McNemar's test)

Bold values indicates statistically significant with $p$-value less than 0.05

$E G$ early glaucoma, $R N F L$ retinal nerve fiber layer, $c P R N F L$ circumpapillary RNFL, GC-IPL ganglion cellinner plexiform layer employed the wide-field SS-OCT scan protocol for parameter measurement [26, 27]. However, unlike the current inaugural investigation, these studies evaluated RNFL thickness measurement using only the wide-field SS-OCT protocol: they did not utilize wide-field RNFL maps. In the present study as in previous studies, we evaluated the continuous parameters' diagnostic abilities between widefield SS-OCT and SD-OCT by AUC (the DeLong method) (Supplementary Table 4) as well as the diagnostic abilities of wide-field RNFL maps themselves. Similarly to the previous studies, there were no differences in the diagnostic abilities of the two OCT devices other than the average GCIPL value. Further, we explored the issue of whether the two devices' parameter differences could lead to a divergence in diagnostic power, but no significant difference was found. Further evaluation is needed to clarify this aspect.

Recently, our group evaluated the diagnostic ability of wide-field SS-OCT RNFL thickness maps for detection of PPG or EG. Whereas the results did show good diagnostic performance, we had evaluated only SS-OCT data [28]. By contrast, in the present study, we directly compared the diagnostic abilities of both SS-OCT and SD-OCT thickness and deviation maps. In PPG and EG diagnoses, the sensitivities of the wide-field SS-OCT maps were comparable to those of most of the SD-OCT thickness and deviation maps and superior to those of the maps showing $p$-values under 0.05 (see Tables 2 and 3). In many cases, close inspection of the SD-OCT RNFL or GC-IPL thickness or deviation map revealed glaucomatous structural changes corresponding to the same areas of RNFL thinning shown by the wide-field SS-OCT RNFL maps. Although close inspection of both the SD-OCT-imaged RNFL and GC-IPL could detect the glaucomatous structural changes well, there are certain advantages of wide-field SS-OCT maps. Specifically, because the SuperPixel map encompasses the macular [GCIPL+RNFL] area, which includes the RNFL, it is much better for visualization of the RNFL defect continuum. Also, whereas combined use of SD-OCT RNFL and GCIPL maps for determination of structural changes is necessarily subjective, wide-field SS-OCT maps offer the capacity for objective visualization.

In some instances in the present cpRNFL thickness and GC-IPL analyses by SS-OCT or SD-OCT, no early structural changes were detected. On the wide-field SS-OCT RNFL maps however, definite RNFL changes could be observed: on the RNFL thickness map as a wedge-shaped dark-blue area surrounded by an abrupt color-scale change, and on the SuperPixel map as a wedge-shaped area of contiguous abnormal yellow/red pixels along with RNFL thinning. (Fig. 1, Supplementary Figure 1 \& 2). Furthermore, in some normal cases determined by conventional red-free photographs and SD-OCT, the wide-field RNFL maps could detect very early structural changes (Fig. 1). In these cases, the diagnosis could be considered to be 
changed from normal to PPG. Indeed, under such circumstances, wide-field RNFL thickness maps could prove very critical in clinical practice.

As a new OCT technology, SS-OCT offers the advantages of high speed, long wavelength and high penetration, which together make possible wide-field visualization with a single-scan protocol. Moreover, with SS-OCT, cpRNFL, and macular-area [GC-IPL+RNFL] analyses are performed simultaneously, which can lower the error rate and reduce the examination time. As such, the wide-field SS-OCT RNFL thickness maps can be an effective supplementary or alternative imaging modality to conventional red-free photography. However, it is important to recognize that there are also limitations to wide-field SSOCT technology. Like other OCTs, layer segmentation can be difficult due to macular diseases such as epiretinal membrane, wet age-related macular degeneration, and macular edema; also, imaging acquisition is potentially interfered with by low signal strengths due to media opacities. Accordingly, we excluded eyes with such conditions from our study.

Our study has several weaknesses. First, we analyzed RNFL defect using only a qualitative method. However, in the clinical setting, the quantitative aspect of RNFL defect, including angular width and area, cannot be measured $[19,33]$. In any event, the main strength of wide-field RNFL maps is fast scan speed and the convenience of the provision of important information in an at-a-glance view. Second, we determined whether the RNFL defect was visible or not, which depends on the physician's subjective judgment; thus, the objectivity of those determinations could be challenged. For the wide-field RNFL thickness map, the criteria for determining "abnormal" may be ambiguous and not objective. Although SuperPixel maps' "abnormal" indication is an objective method, there is not enough information on the thickness threshold of abnormality, which is to say, the device's normative database. According to the above-noted difference between the widefield SS-OCT scan and the conventional scan method or SD-OCT, the normative database for each OCT device or scan protocol also will differ. If an analysis taking account of such difference is performed, the strength of this study's results will be enhanced. Third, the criteria used for PPG have the possibility of incorrect diagnostic classification. Through longitudinal studies in the future, it will be more useful to prove that PPG eye has significant structural progression under our observation rather than only showing glaucomatous structural change.

In conclusion, the wide-field SS-OCT RNFL thickness maps were demonstrated to be effective as well as comparable to conventional SD-OCT RNFL maps for discrimination of early stages of glaucoma from healthy eyes. They can be especially useful as a complementary or alternative modality to conventional imaging tools for detection of early stages of glaucoma.

\section{Summary}

\section{What was known before}

- Wide-field SS-OCT RNFL thickness maps showed the superior glaucoma-diagnostic ability to the conventional criteria.

\section{What this study adds}

- Wide-field SS-OCT RNFL thickness maps were demonstrated to be effective as well as comparable to conventional SD-OCT for discrimination of early stages of glaucoma from healthy eyes.

\section{Compliance with ethical standards}

Conflict of interest The authors declare that they have no conflict of interest.

\section{References}

1. Weinreb RN, Aung T, Medeiros FA. The pathophysiology and treatment of glaucoma: a review. JAMA. 2014;311:1901-11.

2. Hirooka K, Manabe S, Tenkumo K, et al. Use of the structurefunction relationship in detecting glaucoma progression in early glaucoma. BMC Ophthalmol. 2014;14:118.

3. Hood DC, Kardon RH. A framework for comparing structural and functional measures of glaucomatous damage. Prog Retin Eye Res. 2007;26:688-710.

4. Quigley HA, Katz J, Derick RJ, et al. An evaluation of optic disc and nerve fiber layer examinations in monitoring progression of early glaucoma damage. Ophthalmology. 1992;99:19-28.

5. Tuulonen A, Airaksinen PJ, Montagna A, Nieminen H. Screening for glaucoma with a non-mydriatic fundus camera. Acta Ophthalmol. 1990;68:445-9.

6. Sommer A. Retinal nerve fiber layer. Am J Ophthalmol. 1995;120:665-7.

7. Sommer A, Miller NR, Pollack I, et al. The nerve fiber layer in the diagnosis of glaucoma. Arch Ophthalmol. 1977;95:2149-56.

8. Bussel II, Wollstein G, Schuman JS. OCT for glaucoma diagnosis, screening and detection of glaucoma progression. Br J Ophthalmol. 2014;98(Suppl 2):ii15-9.

9. Grewal DS, Tanna AP. Diagnosis of glaucoma and detection of glaucoma progression using spectral domain optical coherence tomography. Curr Opin Ophthalmol. 2013;24:150-61.

10. Vessani RM, Moritz R, Batis L, et al. Comparison of quantitative imaging devices and subjective optic nerve head assessment by general ophthalmologists to differentiate normal from glaucomatous eyes. J Glaucoma. 2009;18:253-61.

11. Jeoung JW, Choi YJ, Park KH, Kim DM. Macular ganglion cell imaging study: glaucoma diagnostic accuracy of spectral-domain optical coherence tomography. Invest Ophthalmol Vis Sci. 2013;54:4422-9.

12. Kim KE, Ahn SJ, Kim DM. Comparison of two different spectral domain optical coherence tomography devices in the detection of 
localized retinal nerve fiber layer defects. Jpn J Ophthalmol. 2013;57:347-58.

13. Kim YJ, Kang MH, Cho HY, et al. Comparative study of macular ganglion cell complex thickness measured by spectral-domain optical coherence tomography in healthy eyes, eyes with preperimetric glaucoma, and eyes with early glaucoma. Jpn J Ophthalmol. 2014;58:244-51.

14. Kim YK, Yoo BW, Kim HC, Park KH. Automated detection of hemifield difference across horizontal raphe on ganglion cell--inner plexiform layer thickness map. Ophthalmology. 2015;122:2252-60.

15. Seong M, Sung KR, Choi EH, et al. Macular and peripapillary retinal nerve fiber layer measurements by spectral domain optical coherence tomography in normal-tension glaucoma. Invest Ophthalmol Vis Sci. 2010;51:1446-52.

16. Ye $\mathrm{C}$, To $\mathrm{E}$, Weinreb $\mathrm{RN}$, et al. Comparison of retinal nerve fiber layer imaging by spectral domain optical coherence tomography and scanning laser ophthalmoscopy. Ophthalmology. 2011;118:2196-202.

17. Leung CK, Lam S, Weinreb RN, et al. Retinal nerve fiber layer imaging with spectral-domain optical coherence tomography: analysis of the retinal nerve fiber layer map for glaucoma detection. Ophthalmology. 2010;117:1684-91.

18. Sakamoto A, Hangai M, Nukada M, et al. Three-dimensional imaging of the macular retinal nerve fiber layer in glaucoma with spectral-domain optical coherence tomography. Invest Ophthalmol Vis Sci. 2010;51:5062-70.

19. Shin JW, Uhm KB, Lee WJ, Kim YJ. Diagnostic ability of retinal nerve fiber layer maps to detect localized retinal nerve fiber layer defects. Eye. 2013;27:1022-31.

20. Hwang YH, Jeong YC, Kim HK, Sohn YH. Macular ganglion cell analysis for early detection of glaucoma. Ophthalmology. 2014;121:1508-15.

21. Mansouri K, Medeiros FA, Tatham AJ, et al. Evaluation of retinal and choroidal thickness by swept-source optical coherence tomography: repeatability and assessment of artifacts. Am J Ophthalmol. 2014;157:1022-32.

22. Mrejen S, Spaide RF. Optical coherence tomography: imaging of the choroid and beyond. Surv Ophthalmol. 2013;58:387-429.

23. Lee SY, Bae HW, Kwon HJ, et al. Repeatability and agreement of swept source and spectral domain optical coherence tomography evaluations of thickness sectors in normal eyes. J Glaucoma. 2016;26:e46-53.

24. Kim MJ, Park KH, Yoo BW, et al. Comparison of macular GCIPL and peripapillary RNFL deviation maps for detection of glaucomatous eye with localized RNFL defect. Acta Ophthalmol. 2015;93:e22-8.

25. Kim YW, Kim DW, Jeoung JW, et al. Peripheral lamina cribrosa depth in primary open-angle glaucoma: a swept-source optical coherence tomography study of lamina cribrosa. Eye. 2015;29:1368-74.

26. Yang Z, Tatham AJ, Weinreb RN, et al. Diagnostic ability of macular ganglion cell inner plexiform layer measurements in glaucoma using swept source and spectral domain optical coherence tomography. PLoS ONE. 2015;10:e125957.

27. Yang Z, Tatham AJ, Zangwill LM, et al. Diagnostic ability of retinal nerve fiber layer imaging by swept-source optical coherence tomography in glaucoma. Am J Ophthalmol. 2015; 159:193-201.

28. Lee WJ, Na KI, Kim YK, et al. Diagnostic ability of wide-field retinal nerve fiber layer maps using swept-source optical coherence tomography for detection of preperimetric and early perimetric glaucoma. J Glaucoma. 2017;26:577-85.

29. Kim TJ, Kim YK, Kim DM. Patterns of subsequent progression of localized retinal nerve fiber layer defects on red-free fundus photographs in normal-tension glaucoma. Korean J Ophthalmol. 2014;28:330-6.

30. Bland JM, Altman DG. Measuring agreement in method comparison studies. Stat Methods Med Res. 1999;8:135-60.

31. Ha A, Lee SH, Lee EJ, Kim TW. Retinal nerve fiber layer thickness measurement comparison using spectral domain and swept source optical coherence tomography. Korean J Ophthalmol. 2016;30:140-7.

32. Lee KM, Lee EJ, Kim TW, Kim H. Comparison of the abilities of SD-OCT and SS-OCT in evaluating the thickness of the macular inner retinal layer for glaucoma diagnosis. PLoS ONE. 2016;11: $\mathrm{e} 0147964$.

33. Kim MJ, Jeoung JW, Park KH, et al. Topographic profiles of retinal nerve fiber layer defects affect the diagnostic performance of macular scans in preperimetric glaucoma. Invest Ophthalmol Vis Sci. 2014;55:2079-87. 